\title{
LA SOLUCIÓN LEIBNIZIANA AL PROBLEMA DE LA RELACIÓN CUERPO-ALMA DESDE UNA PERSPECTIVA ACTUAL ${ }^{1}$
}

\section{THE LEIBNIZIAN SOLUTION TO THE PROBLEM OF THE RELATIONSHIP BODY-SOUL FROM A CURRENT PERSPECTIVE}

\author{
HANS POSER ${ }^{2}$ \\ Technische Universität, Berlin
}

Resumen: El problema de la relación mente-cuerpo, a día de hoy, difiere de aquél del siglo XVII en la medida en que no es tanto discutido como problema ontológico cuanto como cuestión para las ciencias empíricas y, en el mejor de los casos, para la epistemología. Se remonta a Descartes y encontró su solución leibniziana gracias a la armonía preestablecida entre mónadas y sustancias, y entre los cuerpos de éstas como

\footnotetext{
${ }^{1}$ Presento aquí un trabajo (traducido por Roberto R. Aramayo y Concha Roldán) que desarrolla y completa el presentado al Congreso Internacional de la Sociedad española Leibniz Ciencia, tecnología y bien común: La actualidad de Leibniz, celebrado en Valencia entre el 21 y el 23 de marzo 2001 bajo el título ":Qué cabe aprender de la solución leibniziana al problema de la relación alma-cuerpo?" y que fue recogido en las Actas de dicho Congreso (Valencia: Universidad Politécnica 2002). En esta versión desarrollada y más precisa de aquel trabajo tuvieron mucha relevancia las discusiones mantenidas con Quintín Racionero, por lo que me parece de la mayor justicia dedicarle estas páginas en un monográfico concebido como homenaje a su persona.

${ }^{2}$ Profesor Emérito en el Institut für Philosophie, Literatur-, Wissenschafts-und Technikgeschichte, Technische Universität Berlin. E-mail: hans.poser@tu-berlin.de
} 
fenómenos; esto es, como sustancias corpóreas garantizadas de 'arriba a abajo' por la selección divina respecto del mundo. Esto supone ciertas presunciones que serán analizadas en el presente trabajo, a fin de señalar que las propuestas modernas de cara a una solución por medio de complejos procesos evolucionarios depende de una vía 'de abajo a arriba', y se puede someter a crítica por medio de conjeturas comparables.

Palabras Clave: Leibniz, relación mente-cuerpo, cogito,

AвSTRACT: The mind-body problem of today differs from that of the 17 th century, as it is not so much discussed as an ontological one, but as a question of the empirical sciences-at best of epistemology. It goes back to Descartes, and found its Leibnizian solution by the pre-established harmony between Monads as substances, and their bodies as phenomena - namely, corporeal substances—warranted 'top down' by God's selection of the world. This needs presumptions that are analysed here, in order to indicate that modern proposals for a solution via complex evolutionary processes depend on a way that is 'bottom up', yet by introducing comparable conjectures.

KeYwords: Leibniz, relation body-soul, cogito.

\section{Situación del problema}

Rudolf Carnap había publicado en el año 1928 su obra Pseudoproblemas en la filosofía, donde intentaba mostrar que la lucha entre idealismo y realismo carecía de sentido, al no ser posible de ninguna manera demostrar o refutar una de las dos posiciones. Poco después, en los años cincuenta del pasado siglo XX Ludwig Wittgenstein tuvo una gran repercusión al proponer como "solución” la idea de que los problemas de este tipo descansan sobre un hechizo de la mente, el cual se origina por una mezcolanza de diferentes juegos lingüísticos -descansando el uno sobre objetos físicos, el otro sobre sensaciones y estados anímicos. Por su parte, Gilbert Ryle casi convenció a dos generaciones de que hablar de la mente significa tanto como hablar de un fantasma dentro de una máquina y de ahí surgió la unánime apreciación de que la filosofía debería enterrar el antiguo problema de la relación alma-cuerpo. Pese a ello, las últimas décadas certifican un sorprendente renacimiento del problema. Ahora no se trata de la diferenciación que se puede hacer entre "cuerpo animado" (Leib) y "cuerpo físico" (Körper) por un lado y entre "mente" (Geist) y "alma" (Seele) por el otro -estos dos conceptos son manejados con significados equivalentes, cuando no debe subrayarse expresamente que "cuerpo físico" puede concernir también a lo inanimado (verbigracia una piedra) y "alma" puede concernir también a lo inconsciente (verbigracia un 
alma animal); proceder de otra manera significaría generar una absurda diferencia entre el problema "alma-cuerpo" en lengua alemana y el problema angloamericano de "mente-cuerpo" (mind-body) . $^{3}$

Sin duda, el problema actual de la relación alma-cuerpo es muy distinto al del siglo XVII, pues ya no es visto como un problema ontológico, sino como un problema de las ciencias empíricas o, a lo sumo, de la teoría del conocimiento. Los argumentos que se han renovado hoy en día a este respecto proceden de dos ámbitos diferentes; el primero es el criterio operacional de la conciencia señalado por Alan Turing, según el cual a un "X" -ya se trate de un hombre o de una máquina- colocado en una habitación separada ha de serle atribuida conciencia, cuando no cabe averiguar mediante preguntas a ese " $\mathrm{X}$ " desde otra habitación, $\mathrm{y}$ en base a una valoración de sus respuestas, si dicho " $\mathrm{X}$ " se trata de una máquina o de un ser humano. Frente al pensamiento de Turing cabe observar las siguientes secuelas críticas o refutaciones:

a. La moderna fórmula de Hilary Putnam acerca de la mente en la botella, ${ }^{4}$ a saber, el experimento mental del cerebro en un tarro, que es dirigido por un gran ordenador y no está en situación de distinguir entre un mundo externo real y sus sensaciones internas -una concepción que, por una parte, desemboca en una especie de funcionalismo y, por la otra, en una ontología platónica de las matemáticas.

b. La habitación china de John Searle, ${ }^{5}$ en la que a través de una hendidura se van mostrando signos chinos que, sin ser comprendidos por los seres humanos que se hallan en la habitación, son "respondidos" a partir de una lista dada de antemano con signos ordenados -un experimento mental que debe mostrar que es imposible reducir la semántica a la sintaxis, siendo esta última la única que puede ser programada en una máquina. Por ello, según Searle, es imposible una reducción tal, esto es, un reduccionismo de lo mental a lo material. De manera similar argumenta Roger Penrose con referencia a la necesidad de un platonismo mínimo en las matemáticas. ${ }^{6}$

\footnotetext{
${ }^{3}$ N.T.: Hemos optado por conservar entre paréntesis los términos originales, pues el castellano no distingue y precisa los significados lo mismo que el alemán, y para que se vea la terminología usada en el mundo anglosajón.

${ }^{4}$ Hilary Putnam: Reason, Truth and History, Cambridge: Cambridge UP 1981, c. 1.

${ }^{5}$ John Searle: Minds, Brains, and Programs, en: The Behavioral and Brain Sciences, 1980 (3), 417-457.

${ }^{6}$ Roger Penrose: The Emperor's New Mind: Concerning Computers, Minds and The Laws of Physics, Oxford: Oxford UP 1989.
} 
c. Umberto Maturana y Francisco Varela representan el denominado contructivismo radical, ${ }^{7}$ que saca las consecuencias ontológicas del funcionalismo y rechaza absolutamente la existencia de un mundo externo a mi conciencia.

Toda esta discusión gira en torno a la cuestión de la posibilidad de una inteligencia artificial, a cuyo efecto la mayoría sostiene una posición materialista, llevados por el convencimiento de que sólo es una cuestión de tiempo el que todas las funciones de la mente puedan ser simuladas mediante un ordenador.

La segunda manera en que se aborda la polémica en torno al problema de la relación alma-cuerpo deriva de la neurobiología y queda caracterizada por las siguientes posiciones:

a. El neurobiólogo Paul M. Churchland defiende un materialismo eliminativo, ${ }^{8}$ el cual aspira a evidenciar como superflua toda expresión sobre lo mental, mientras

b. los neurofisiólogos hacen suyos los argumentos de Searle y Penrose adaptándolos a su objetivo de un materialismo funcional, para mostrar que en particular con el trasfondo del problema de la intencionalidad resulta imposible reducir las expresiones mentalistas, tal como se emplean en la psicología, a expresiones no mentalistas.

También aquí las variantes del materialismo -eliminativo, reductivo, funcional y relativo a las teorías de la interpretación- suponen las posiciones más frecuentes. Esto se manifiesta en que Ryle fue un exorcista sumamente eficaz, cuando interpretó el término "mente" como un "fantasma", para desterrarlo. Sin embargo, la actual polémica muestra en todos los casos que el viejo problema de la relación alma-cuerpo, que fuera considerado como una cuestión metafísica sin sentido, está todo lo más muerto sólo en apariencia. Mientras tanto, se están defendiendo enfoques no reduccionistas, comenzando con:

${ }^{7}$ Humberto Maturana, Francisco Varela: El árbol del conocimiento, Santiago de Chile: Ed. Universitaria 1984.

${ }^{8}$ Paul M. Churchland: Matter and Consciousness. Cambridge: PIT Press 1984. 
a. los argumentos a favor de un antireduccionismo, tal como son expuestos verbigracia por Thomas Nagel, ${ }^{9}$ cuando muestra que yo nunca estaré en situación de saber en qué consiste ser un murciélago; más aún:

b. la propuesta de Nicholas Rescher, de que ambas partes, cuerpo y mente, sean vistas como procesos, ${ }^{10}$ de manera que en la concepción del proceso coincidan juntas operaciones físicas, bióticas, psíquicas y mentales; y finalmente:

c. el enfoque de una Monadología cuántica (Quantenmonadologie) de Teruaki Nakagomi ${ }^{11}$, completamente apoyada en el pensamiento de Leibniz, y que por el lado de la conciencia propone un significado teórico cuántico, mientras que por el lado material lo que sugiere es una interpretación causal.

Resulta particularmente llamativo que durante las últimas décadas la discusión se haya dirigido hacia un nuevo tipo de solución y con ello hacia una nueva variante del materialismo, el llamado materialismo no reductivo, que se caracteriza por la idea de un sobrevenimiento o emergencia. Con el trasfondo de las conjeturas teórico-evolutivas de la biología, las teorías de la emergencia intentan explicar cómo pudo ocurrir que la materia se transfiriese a un biosistema de autoconservación, que los biosistemas desarrollasen cualidades sensibles y se transformasen en psicosistemas, a la par que finalmente los psicosistemas pretendían adquirir las nuevas cualidades de la reflexión -cualidades todas ellas no reducibles a cualidades meramente materiales. Este renacimiento del problema de la relación almacuerpo arroja una nueva luz sobre el planteamiento leibniziano y su solución.

\footnotetext{
9 Thomas Nagel: What Is it Like to be a Bat? En: The Philosophical Review 83.4 (1974) 435450 .

${ }^{10}$ Nicholas Rescher: Process Philosophy and Monadological Metaphysics, en: Monadisches Denken in Geschichte und Gegenwart, hg. von Sigmund Bonk, Würzburg: Königshausen \& Neumann 2003, 209-217.

11 Teruaki Nakagomi: Quantum Monadology: A World Model to Interpret Quantum Mechanics and Relativity. En: Open Systems \& Information Dynamics 1.3 (1992) 355-378.
} 


\section{Descartes y la dificultad sistemática del problema de la relación alma-cuerpo}

Desde que Descartes escindió radicalmente la res cogitans y la res extensa, el problema de la relación alma-cuerpo quedó establecido como uno de los desafíos centrales de la modernidad, pues ¡cómo es posible que concuerden ambas facetas? Peter Bieri ha formulado el problema en forma de tres enunciados, enunciados que parecen aceptables tomado cada uno por separado, si bien no son conciliables entre sí: ${ }^{12}$

1) Los fenómenos mentales son fenómenos no-físicos (la mente es inmaterial).

2) Los fenómenos mentales pueden causar fenómenos físicos (la mente opera sobre el cuerpo, o sea, origina una diferencia en el mundo material).

3) El ámbito de los fenómenos físicos (esto es, el mundo material o corporal) es cerrado de manera causal.

El primer enunciado expresa nuestra convicción cotidiana de que mente y cuerpo pertenecen a dos ámbitos ontológicos distintos que se hallan claramente escindidos: el amor, el miedo, el pensar y la voluntad pertenecen al primero; la extensión, el peso, el calor y la impenetrabilidad al segundo. Así pues, este enunciado pone de manifiesto el dualismo ontológico.- El segundo enunciado implica la bien conocida experiencia de un puente tendido entre ambos ámbitos, como temblar de miedo, sonrojarse de indignación o hacer algo intencionadamente.Por último, el tercer enunciado tiene su origen en la concepción de la física del siglo XVII, según la cual la naturaleza es comprendida como materia y no como algo animista, como se entiende en la alquimia: los fenómenos físicos son sólo causados por fenómenos físicos y explicar un fenómeno físico requiere una explicación basada en condiciones físicas y leyes naturales nada más: un experimento no depende de fórmulas mágicas (o conjuros). Bieri ha llamado a este enunciado fisicalismo metodológico. En realidad, el éxito de las ciencias naturales modernas descansa sobre este presupuesto.

Estos tres enunciados son susceptibles de aprobación tomados por separado, pero son incompatibles en tanto que tomados dos a dos contradicen al tercero. Eso es justo lo que ocasiona el problema de la relación alma-cuerpo. Ahora bien,

\footnotetext{
${ }^{12}$ Bieri, Peter: Generelle Einführung, en: P. Bieri. (ed.), Analytische Philosophie des Geistes, Bodenheim: Athenäum ${ }^{2} 1993,1-28$, p. 5.
} 
Descartes ensayó una solución para conciliar entre sí los tres enunciados, al seguir un camino que tradicionalmente es tomado de una exposición leibniziana de Descartes. En el contexto que aquí consideramos es irrelevante si Descartes defendió la posición que luego le atribuyó Leibniz, tal como piensan la mayoría de los investigadores cartesianos, o no (como algunos intentan mostrar). Lo que a mí me interesa es la solución leibniziana y esta a su vez desde el trasfondo de la actual discusión entre neurobiólogos, partidarios de una inteligencia artificial y filósofos.

Partamos, pues, de la reconstrucción y crítica leibnizianas de Descartes. En el $\$ 80$ de su Monadología Leibniz escribe: "Descartes ha reconocido que las almas no pueden transferir fuerza alguna a los cuerpos, porque siempre hay la misma cantidad de fuerza en la materia. Creyó, sin embargo, que el alma podía cambiar la dirección de los cuerpos". Esto hay que entenderlo de la siguiente manera: Descartes creía que la voluntad podía modificar en el interior de la glándula pineal, de la epífisis, la dirección del movimiento de los spiritus animales de los nervios. Merced a ello intentó hacer compatibles los tres enunciados de Bieri: partiendo de un dualismo ontológico como el expresado en (1), Descartes desarrolla una peculiar forma del influjo entre la voluntad y los spiritus animales de los nervios y con ello está de acuerdo con (2); y finalmente aboga por (3), porque este peculiar tipo de influjo no destruye la unidad de la física (como un sistema cerrado causal), al ser compatible con la ley del mantenimiento de la fuerza. Pero Leibniz se opone a esto: "La razón de ello fue que en su época no se conocía la ley natural que establece también la conservación de la misma dirección total de la materia. Si [Descartes] hubiese advertido esto, habría venido a parar a mi sistema de armonía preestablecida" ${ }^{13}$. Así pues, Leibniz muestra que una ley física, a saber, la conservación del impulso como un vector, hace imposible adherirse al enunciado (2).

Para decirlo con Peter McLaughlin, rechazó Leibniz (2), para solucionar el problema de la relación alma-cuerpo, rechazando por tanto el influjo de la voluntad sobre el mundo; en cambio Hobbes negaba (1), o sea, la inmaterialidad de la mente, mientras que hoy rechazamos al menos a (3) la tesis de la unidad

${ }^{13}$ „Des Cartes a reconnu, que les Ames ne peuvent point donner de la force aux corps, parce qu'il y a tousjours la même quantité de force dans la matiere. Cependant il a crû, que l'ame pouvoit changer la direction des corps. Mais c'est par ce qu'on n'a point sû de son temps la loy de la nature, qui porte encor la conservation de la même direction totale dans la matiere. S'il l'avoit remarquée, il seroit tombé dans mon Systeme de l'Harmonie préétablie. " Monadología, $\$ 80$. 
causal del mundo material. ${ }^{14}$ Dediquemos nuestra atención tras estos preámbulos a la solución leibniziana.

\section{La solución leibniziana: la armonía preestablecida}

La pregunta de Descartes sobre la relación entre res cogitans y res extensa, el ocasionalismo de Malebranche y el monismo de Spinoza configuran los antecedentes de la solución leibniziana al problema de la relación alma-cuerpo. Precisamente en la polémica con Spinoza queda claro que para Leibniz no se trata de un mero problema ontológico, sino de hacer posible la libertad. Un determinismo que contemple estas dos facetas, la res cogitans y la res extensa, como plenamente acopladas en el sentido de Spinoza, excluiría por completo a la libertad -y con ella a la posibilidad de una ética y de un obrar responsable. Se trata de que tengamos esto siempre presente en lo que sigue, cuando se trata de la relación entre monadas, cuerpos y sustancias corporales.

La respuesta de Leibniz, la armonía preestablecida, fue asumida con reticencia por Christian Wolff, criticada por Johan Christoph Gottsched y rechazada por Kant. Su tesis central de un "paralelismo perfecto" de cuerpo y alma, ${ }^{15}$ expresada reiteradamente con la metáfora de dos relojes sincronizados, es bien conocida y no necesita verse repetida aquí. Lo que sí resulta de interés es el fundamento de la solución leibniziana, su valoración de ambas facetas y sus argumentos a favor del paralelismo -si la metáfora es en todo caso adecuada, pues presupone un dualismo de dos ámbitos ontológicos diferentes y equivalentes que se hallan en una armonía preestablecida. Al fin y al cabo, uno de los principales puntos de partida de la metafísica leibniziana es, como es sabido, su rechazo del materialismo avant la lettre. Sin presentar de modo exhaustivo su concepción, señalemos como significativo que todos los argumentos leibnizianos contra el carácter

\footnotetext{
${ }^{14}$ Peter McLaughlin: "Descartes on Mind-Body Interaction and the Conservation of Motion”, en: The Philosophical Review 102 (1993) 155-182; p. 158.

15 „J'ay etabli un parallelisme parfait entre ce qui passe dans l'ame et entre ce qui arrive dans la matiere, ayant monstré, que l'ame avec ses fonctions est quelque chose de distinct de la matiere, mais que cependant elle est tousjours accompagnée des organes de la matiere, et qu'aussi les fonctions de l'ame sont tousjours accompagnées des fonctions des organes, qui leur doivent repondre, et que cela est reciproque et le sera tousjours." Considerations sur la doctrine d'un Esprit Universel Unique, GP VI 533.
} 
sustantivo de la materia descansan sobre presupuestos metafísicos, como sobre el concepto de sustancia como un Unum per se, sobre el principio de continuidad con la consecuencia de una divisibilidad discrecional, sobre la coincidencia del pensar y el ser, así como sobre el principio de conservación de la vis. Todos ellos parecen excluir no sólo al cuerpo en el sentido de un dualismo ontológico, sino también a una "sustancia corporal" entendida como una unidad de alma y cuerpo. Sin embargo, Leibniz utiliza justamente este concepto tan a menudo que la unión entre cuerpo y mente ha de ser vista de tal modo que asegure que los argumentos contra el materialismo no destruyan al mismo tiempo la posibilidad de una sustancia corporal.

Ahora bien, la ontología leibniziana es mucho más rica que un estricto idealismo, pues abarca:

- Ideas, desde ideas simples sobre el concepto completo de una sustancia individual hasta los mundos posibles en la Regio idearum,

- monadas con percepciones y apetitos hasta el mundo de las monadas,

- cuerpos materiales que pertenecen el mundo de la naturaleza hasta todo el universo.

Según la Monadología los cuerpos son agregados de sustancias (Mon. \$2) y al mismo tiempo, como escribe en una carta a Rémond, "sólo fenómenos bien fundados". Además, cuerpo y alma están tan perfectamente separados que el influjo de una faceta sobre la otra sólo puede ser “ideal” (Mon. \$51). Por eso este tipo de influjo ideal o de relación interna es el que ha de llevar la carga de la conexión entre la unidad sustancial de las mónadas y el cuerpo al cual pertenecen.

La libertad, la voluntad libre como problema central dentro del más amplio de la relación alma-cuerpo, es un peculiar e importante campo de este influjo ideal: una acción es libre, si resulta de una decisión racional al sopesar las posibilidades entre acciones alternativas conforme al principio de lo mejor. Esta decisión es parte de la ley interna de una mónada dotada de razón -con ello no presupone sólo el propio "concepto consumado de la sustancia individual" que contiene el transcurso vital, también reclama tanto la sintonía de todos los conceptos individuales de este mundo tomados uno por uno, como la supresión de otras posibilidades, esto es, de otros mundos posibles: la libertad fuerza este amplio margen. 
El concepto completo de la sustancia individual, al igual que la relación interna, suele verse reducido a la construcción formal de mundos lógicos en el reino de las ideas. También esta fundamentación es bien conocida y supone un primer paso, muy importante, en la fundamentación; pero es insuficiente para comprender qué son las sustancias corporales. Leibniz define una "sustancia corporal" como algo "que consiste en una sustancia simple o mónada (esto es, en un alma o algo análogo al alma) y un cuerpo orgánico que está unido a ésta”. ${ }^{16}$ En una carta a Volder ofrece Leibniz su famosa quíntuple respuesta a la pregunta sobre su concepto de sustancia:

"Por tanto, yo distingo entre:

1. la entelequia primitiva o alma,

2. la materia, a saber, la materia prima o fuerza pasiva primitiva,

3. la mónada íntegra, en la que están unidos ambos momentos,

4. la masa o materia segunda, esto es, la máquina orgánica en donde concurren innumerables mónadas subalternas,

5. el animal o la sustancia corporal, que obtiene su unidad por medio de la mónada dominante en la máquina”. ${ }^{17}$

"Entelequia primitiva significa aquí fuerza activa primitiva, la cual ha de ser considerada, al igual que la "fuerza pasiva primitiva", como una propiedad de la mónada. Incluso si por ende "el cuerpo es un agregado de sustancias, pero

\footnotetext{
16 „Substantiam corpoream voco, quae in substantia simplice seu monade (id est anima vel Animae analogo) et unito ei corpore organico consistit." Carta a Bierling, 12 Aug. 1711.

17 „Distinguo ergo (1) Entelechiam primativam seu Animam, (2) Materiam nempe primam seu potentiam passivam primitivam, (3) Monada his duabas completam, (4) Massam seu materiam secundam, sive Machinam organicam, ad quam innumerae concurrunt Monades subordinatae, (5) Animal seu substantiam corpoream, quam Unam facit Monas dominans in Machinam." En Correspondencia con De Volder, 20 June 1703, GP II 252.
} 
estrictamente no es ninguna sustancia", ${ }^{18}$ como Leibniz escribe a Arnauld, si bien cada sustancia está "dotada de un cuerpo". ${ }^{19}$

\section{De la idea al cuerpo: fuerzas y emanación}

Lo que falta todavía es la conexión interna y no-lógica entre (i) ideas como construcción lógica del concepto completo de sustancias individuales, (ii) mónadas como unidades de actividad interna con percepciones, (iii) cuerpos vivos y activos que contienen una mónada, y (iv) simples agregados que siguen leyes físicas en sus movimientos. Leibniz da una doble respuesta: que ha de ser considerada como las dos caras de una moneda, al recurrir por un lado al concepto de fuerza y por el otro al concepto de emanación.

El concepto de fuerza es introducido por Leibniz en su Dinámica. Tiene una vertiente física; las fuerzas en el ámbito de los cuerpos son simplemente de naturaleza derivativa. En cambio, las fuerzas primitivas han de tener su fuente en las sustancias como "fuerza activa", "que contienen un tipo de actividad o entelequia". ${ }^{20}$ En Specimen dynamicum Leibniz muestra que "fuerza activa", en su terminología vis activa primitiva, "es inherente a cada sustancia corporal como tal", mientras que la vis activa derivativa "a partir de la limitación -por así decir- de la fuerza primitiva, resultante de la colisión de los cuerpos entre sí, se presenta de diversas formas". ${ }^{21}$

18 „Le corps est un aggregé de substances, et ce n'est pas une substance à proprement parler. “ En Carta a Arnauld, 23 de marzo de 1690, GP III 135.

${ }^{19}$ Como escribe Adams. Véase: Adams, Robert Merrihew: Leibniz. Deist, Theist, Idealist, New York - Oxford: OUP 1994, p. 264.

${ }^{20}$ "Cujus rei ut aliquem gustum dem, dicam interim, notionem virium seu virtutis (quam Germani vocant Krafft, Galli la force) cui ego explicandae peculiarem Dynamices scientiam destinavi, plurimum lucis afferre ad veram notionem substantiae intelligendam. Differt enim vis activa a potentia nuda vulgo scholis cognita, quod potentia activa Scholasticorum, seu facultas, nihil aliud est quam propinqua agendi possibilitas, quae tamen aliena excitatione et velut stimulo indiget, ut in actum transferatur. Sed vis activa actum quendam sive entelecheian continet, atque inter facultatem agendi actionemque ipsam media est, et conatum involvit; atque ita per se ipsam in operationem fertur; nec auxiliis indiget, sed sola sublatione impedimenti." De primae philosophiae Emendatione, et de Notione Substantiae, GP IV 469.

${ }^{21}$ „Duplex autem est Vis Activa, nempe ut primitiva, quae in omni substantia corporea per se inest, aut derivativa, quae primitivae velut limitatione, per corporum inter se conflictus resul- 
Ahora bien, esto es de gran interés para nuestra cuestión, tal y como continúa: "El uno primitivo (que no es sino la primera entelequia) corresponde al alma o forma substancial; pero justamente por ello no pertenece a las causas generales, las cuales no pueden ser suficientes para explicar los fenómenos" 22 . Dos puntos han de ser resaltados aquí, (i) la atribución de una fuerza primitiva a la forma substancial, al alma y a la sustancia corpórea al mismo tiempo, y (ii) la diferencia evidente entre alma y fenómenos, en tanto que estos últimos son vistos en conexión con causas y fuerzas derivativas. Esto se corresponde con la quíntuple caracterización de la sustancia ofrecida a de Volder, lo que se mantiene también para el poder pasivo, para lo que Leibniz distingue entre fuerzas primitivas y derivadas, que se corresponden con la prima y la secunda materia. $\mathrm{Al}$ hacer un sumario de dichos puntos en la parte II de su Specimen Dynamicum, declara Leibniz que: "A partir de ahí, arrojaremos nueva luz algún día sobre la explicación de la unión del cuerpo y el alma"23. Esto viene a mostrar cuán central es el concepto de fuerza para nuestro problema.

Sin indicarse cómo se procura la conexión entre fuerzas primitivas y derivativas, resulta claro sin embargo que Leibniz ve en ellas el elemento vinculante entre mónadas y cuerpos.

Otro tipo de fuerza, evidentemente diferente, que ha de tomarse en cuenta aparece tras la llamada dinámica de las ideas de Leibniz. Concierne al puente tendido desde el reino de las ideas al mundo de las mónadas. Se puede añadir a la pura construcción lógica de los mundos posibles de dos maneras diferentes. El primer camino consiste en atribuir a las posibilidades una actividad; Leibniz emplea la expresión "existiturire" como un modo de presión de las posibilidades lógicas para ser realizadas, ${ }^{24}$ para lo que describe a las posibilidades como algo que gracias a un "conatus" se encuentra en el camino que lleva de la posibilidad

tans, varie exercetur.“ Specimen dynamicum, pt. I, GM VI 236.


mae substantiali respondet, sed vel ideo non nisi ad generales causas pertinet, quae phaenomenis explicandis sufficere non possunt." Specimen dynamicum, pt. I, GM VI 236.

23 „Ostendimus igitur in omni substantia vim agendi et, si creata sit, etiam patiendi inesse, extensionis notionem per se non completam esse, sed relationem ad aliquid quod extenditur cujus diffusionem sive continuatam replicationem dicat, adeoque substantiam corporis quae agendi resistendique potentiam involvit et ubique massa corporea existit praesupponi, huiusque diffusionem in extensione contineri. Unde aliquando lucem quoque novam explicandae corporis animaeque unioni accedemus." Specimen dynamicum, pt. II, GM VI 247. ${ }^{24}$ „Omne possibile exigit existiturire“, GP VII.289. Véase De ratione cur haec existant potius quam alia, A VI 4,1634 y ss. 
lógica a la realidad, como algo con el afán de existir, y ciertamente dependiendo del respectivo grado de realitas sive perfectio que cada realidad posee. ${ }^{25}$ Esto significa atribuir al dynaton lógico un tipo de entelecheia en sentido aristotélico, que impulsa a los elementos posibles del reino de las ideas a existir como formas activas sustanciales.

El segundo camino se basa en la convicción de que las ideas no actúan por sí mismas, rechazando, por tanto, la identificación de posibilidad lógica con potencialidad. Así, escribió Leibniz criticando a Spinoza: "Las ideas no actúan. Actúa la mente". Esta observación no es un caso aislado, provocado por Spinoza, ${ }^{26}$ sino que su contenido se encuentra repetido en muchas otras ocasiones. Así, en su Diálogo entre Theophile y Polidore de la misma época leemos que: "Las posibilidades carentes de existencia no poseen la fuerza para exixtir hacerse a sí mismas." ${ }^{27}$

Leibniz escribe así dos años después a de Volder: "Una idea es -por así deciralgo muerto e inmutable en sí, como la forma, mientras que el alma es algo vivo y activo, y en este sentido yo no sostengo que sea una idea la que tiende por sí misma al cambio". ${ }^{28}$ La actividad que estamos buscando no descansa pues en la idea, sino en la mente, lo que en relación con los mundos posibles viene a significar en la mente de Dios: "La mente divina es la causa del mundo", escribe en su Discusión con Wagner. ${ }^{29}$

Es Dios quien selecciona dichas posibilidades como candidatos para la creación, las cuales en tanto posibilidades contienen el máximo grado de realitas sive perfectio, no como su entelequia interna, sino a modo de su propia evaluación y elección. Esta interpretación concuerda con la cita del 'existiturire', en la que introduce Leibniz este neo-latinajo por medio del comentario por el cual "[Dios] o el Ser Necesario es la causa en razón de la cual algo existente es mejor que algo

\footnotetext{
25 „ut omne possibile habeat conatum ad Existentiam“, GP VII 289.

${ }^{26}$ - e.g. „Ideae sunt aliquid mere abstractum ut numeri et figurae nec agere possunt“, F. Réf. 44.

27 „Mais les choses possibles n'ayant point d'existence n'ont point de puissance pour se faire exister", Grua 286 / A VI 4, 2232.

28 „Idea est aliquid ut sic dicam mortuum et in se immutabile, ut figura, anima vero aliquid vivum et actuosum, et hoc sensu non dico esse unam aliquam ideam, quae ex se ad mutationem tendat", a de Volder, 23 junio 1699, GP II 184.

29 „Mens divina est causa mundi“, Discusión con Gabriel Wagner, 3 de marzo de 1698, Grua 397.
} 
no existente" ${ }^{30}$; siendo esto apoyado por una reflexión leibniziana en relación al elemento que Kant echará en falta y respecto al cual éste -criticando a Wolffentenderá que no tiene sitio en la fundación de una metafísica sobre la base de posibilidades conceptuales tan solo: a saber, nos referimos al complementum possibilitatis. Leibniz claramente expone en su Nuevo Sistema que tal complemento, que produce el tránsito de la posibilidad a la actualidad, no es de naturaleza conceptual sino el fiat divino ${ }^{31}$. Ese acto inmediato e inmaterial de Dios, es el verdadero fundamento de la vis primitiva activa de cada una de las mónadas, y halla su expresión como el apetito que conduce a las mónadas de percepción a percepción, mientras que el cambio en el mundo corporal acontece de manera que la suma de esas fuerzas permanece constante en el mundo.

Resumiendo, con ello se presenta el cuadro siguiente: la armonía preestablecida depende de algunas premisas metafísicas esenciales, que contienen posibilidades y potencialidades, las cuales permiten desarrollar un orden no-reduccionista. Dicho orden tiene su punto de partida en estructuras lógicas, se enriquece por medio de valores y fuerzas, conduce al reino de las mónadas como sustancias, está presente en un perspectivismo en el nivel de las sustancias corpóreas y alcanza hasta los agregados de sustancia contingentes como materia. En este nivel se presentan nuevas cualidades, que se basan en transformaciones:

- desde las condiciones lógicas de un sujeto hasta la sustancia como un yo,

- desde las percepciones a los fenómenos,

- desde las leyes individuales de cada mónada individual hasta las leyes naturales en el mundo corporal,

- desde el apetito de la mónada hasta el conatus de los cuerpos orgánicos,

- desde la fuerza activa primitiva (vis primitiva activa) de las formas sustanciales hasta la fuerza derivativa activa (vis derivativa activa) de lo orgánico y, finalmente, de todos los cuerpos, y

- desde la fuerza primitiva pasiva (vis primitiva passiva) hasta la masa.

30 „[Deus] Est ergo causa cur Existentia praevalet non-Existentiae, seu Ens nesessarium est Existentificans. " GP VII 289.

31 „l'acte ou le complement de la possibilité“, Système Nouveau, GP IV 479. 
Todas estas transformaciones han de ser concebidas de modo neoplatónico como una emanatio de Dios por medio de las almas hasta los cuerpos orgánicos y, más hacia abajo, hasta la materia. "Emanación" no es ningún término técnico de la filosofía leibniziana de madurez, pero lo utiliza con frecuencia.

De este modo culmina la Arquitectónica de Robinet en un capítulo sobre "La ciencia de la visión: creación y emanación" 32 . En una de sus más tempranas listas de definiciones escribe Leibnit: "La Causa Eficiente es una causa fundada en acción. (La Causa por emanación) es causa eficiente sin alteraciones de si" ${ }^{33}$. Es significativo en grado sumo que Leibniz no use el concepto prácticamente en sitio alguno de forma coloquial, sino que lo use de manera técnica y además en conexión con la creación divina.

Así lo menciona tres veces en el Discurso de Metafísica, el texto clave, en el que se encuentran otros conceptos centrales como el de noción completa de una sustancia individual, el concepto de la forma sustancial y el concepto de sustancia corporal. Leibniz escribe: "En primer lugar, está muy claro que las sustancias creadas dependen de Dios, que las conserva e incluso las produce continuamente por una especie de emanación, como nosotros producimos nuestros pensamientos". ${ }^{34}$

Y algunas páginas después, continúa: "Uno reconoce con claridad que el resto de sustancias depende de Dios del mismo modo que el pensamiento emana de nuestra sustancia" ${ }^{35}$. Este segundo tipo de emanación, esto es, nuestro pensamiento, tiene su fuente en Dios igualmente, como Leibniz escribe a Sophie: "El mismo Dios, que es la fuente de todo bien, es así el fundamento (principe) de todo nuestro conocimiento. Esto tiene validez en la medida que la idea de Dios incluye a la del Ser absoluto, que por así decir equivale a eso que es absolutamente simple en nuestros pensamientos, a partir de lo cual todo lo que comprendemos

\footnotetext{
${ }^{32}$ André Robinet: Architectonique disjonctive, automates systémiques et idéalité transcendentale dans l'oevre de G.W. Leibniz, Paris: Vrin 1986, pp. 418-442.

33 „CAUSA EFFICIENS est causa per actionem. (Causa per emanationem) est causa efficiens sine mutatione sui.“ Ca. 1671, A II 1, 490.

34 „Or il est premierement tres manifeste que les substances créées dependent de Dieu qui les conserve et même qui les produit continuellement par une maniere d'emanation, comme nous produisons nos pensées. “ Discurso de Metafísica $\$ 14$ / A VI 4, 1549.

35 „on voit fort clairement que toutes les autres substances dependent de Dieu comme les pensées emanent de nostre substance“, Discurso de Metafísica $\$ 32$ / A VI 4, 1580.
} 
toma su origen"36. En su Monadología Leibniz usa el término fulguración en ese mismo sentido ${ }^{37}$; pero a nuestro entender, al no aparecer mencionado en ese uso en parte alguna más de la obra de Leibniz, uno podría tomarlo simplemente como sinónimo de emanatio ya que ambas expresiones son empleadas de manera sinónima dentro de la tradición neoplatónica.

No sólo es iluminador aquí el concepto de emanación o fulguración, sino a la misma vez dos cualidades adicionales que Leibniz le atribuye: en primer lugar, el paralelismo que se dibuja entre el pensamiento divino y el pensamiento de los seres humanos como clase de la emanación, y, en segundo lugar, que dicha clase de emanatio es la última fuente de la continuidad: Leibniz habla de una creatio continua como una "emanación continua" en el Discurso de Metafísica, en carta a Bayle ${ }^{38}$, y como "fulguración continua" en la Monadología.

Esto está en consonancia con la afirmación "Ideae non agunt. Mens agit", que muestra la fuente de la dinámica de la Creación, la cual, como algo continuo, garantiza la continuidad de todas las fuerzas, las primitivas y las derivativas, y que establece una jerarquía ontológica de Dios hacia abajo. En el así llamado Sistema Teológico, escrito el mismo año que el Discurso de Metafísica, Leibniz repite esta vinculación al decir que algunos "conciben a Dios como una clase de vis elevada de la cual todo emanaría" ${ }^{39}$. En una pequeña obrita sobre Malebranche escrita 20 años después -es decir, tras 1706- podemos observar una posición similar

\footnotetext{
36 „Dieu qui est la source de tous les biens, est aussi le principe de toutes les connoissances. C'est parce que l'idée de Dieu renferme en elle l'Estre absolu, c'est à dire ce qu'il y a de simple en nos pensées, dont tout ce que nous pensons prend son origine." A Sophie (?), GP IV.292.

37 „Ainsi Dieu seul est l'Unité primitive, ou la substance simple originaire, dont toutes les Monades creées ou derivatives sont des productions, et naissent, pour ainsi dire, par des Fulgurations continuelles de la Divinité de moment à moment, bornées par la receptivité de la creature, à laquelle il est essentiel d'être limitée. "Monadologia, $\$$ 47. Para un análisi adicional de 'fulguratio', véase André Robinet: "Fulgurationen", en: 25 Jahre Gottfried-Wilhelm-Leibniz-Gesellschaft, Hannover 1992, pp. 27-39, donde Robinet muestra, que Leibniz utiliza 'création' y 'émanation' como combinando por un 'o' en el sentido de un 'y', de forma que la emanación, y a partir de ella la fulguración, no debe ser entendida como una forma de alucinación de Dios, o como algo menos real, sino como una analogía, la cual indica la realidad de la sustancia creada en su relación con el creador original e increado (p. 34).

${ }^{38}$ „Dieu, de qui tous les individus emanent continuellement“, Discurso de Metafísica \$14 / A VI 4, 1550sq. - „l'auteur des choses, de qui toutes les realités ou perfections emanent tousjours par une maniere de creation continuelle“, Carta a Bayle, GP III 58.

39 "qui Deum concipiunt tanquam vim quandam summam a qua cuncta quidem emanent", Systema Theologicum, A VI 4, 2357.
} 
en la que "la emanación inmediata y continua" incluye "todas las perfecciones" ${ }^{40}$. Asimismo, en sus notas sobre el primer libro del Ensayo de Locke Leibniz usa el término 'emanar' con respecto a Dios como una sustancia ${ }^{41}$. Y en su muy condensada afirmación acerca de su concepto de mónada dentro de sus comentarios sobre Bayle, deja claro Leibniz que una emanación a partir de los seres humanos como "imitación de lo divino" es un tipo de segunda emanación, regresando en esto a la primera que surge de Dios: "Él es el más elevado centro a partir del cual surge todo por emanación, y si es que algo emana de nosotros al exterior, no sucede de inmediato, sino como él quiso que las cosas estuvieran dispuestas en el exterior y de acuerdo con sus deseos" ${ }^{42}$. Todos estos aspectos vienen a confluir en una carta a Morell de 1698, en la que Leibniz le expone que, en lo que a una acción se refiere, para Dios del mismo modo que para cualquier otra mente, tres formas han de unirse: "fuerza, conocimiento, y voluntad"; y prosigue: "La más elevada esencia de cada sustancia consiste en esta fuerza; es esta fuerza en Dios la que hace que Dios exista con necesidad y que todo lo que existe haya de emanar" ${ }^{43}$. En su intensa discusión con Gabriel Wagner, Leibniz bosqueja la base conceptual de sus nociones de posibilidad y de sustancia, concluyendo que: "Esta serie de posibilidades actuales o mundo emana de Dios sin necesidad, lo que significaría que otras series no serían posibles sino con la certeza y determinación

\footnotetext{
40 „Et qu'il est vray cependant que Dieu nous donne tout ce qu'il y a de positif en cela, et toute perfection y enveloppée par une emanation immediate et continuelle, en vertu de la dependance que toutes les creatures ont de luy, et c'est par là qu'on peut donner un bon sens à cette phrase que Dieu est l'objet de nos ames, et que nous voyons tout en luy." GP VI 578 / A VI 6, 557ss. Véase también la carta a Bayle citada en la nota 37.

${ }^{41}$ „Et comme on peut dire que c'est une verité des plus manifestes, qu'une substance dont la science et la puissance sont infinies, doit estre honnorée, on peut dire qu'elle emane d'abord de la lumiere qui est née avec nous, pourveu qu'on y puisse donner son attention." GP V.23. „l'Estre parfait qui est la derniere raison des choses, et de ses emanations“, Grua 580.

${ }^{42}$ „Mais qu'il y a en Dieu non seulement la concentration, mais encore la source de l'univers. Il est le centre primitif dont tout le reste emane, et si quelque chose emane de nous au dehors, ce n'est pas immediatement, mais par ce qu'il a voulu accommoder d'abord les choses à nos desirs." Extrait du Dictionnaire de M. Bayle, GP IV 553.

${ }^{43}$ "Je serois plustost pour ceux qui reconnoissent en Dieu comme en tout autre esprit trois formalités: force, connoissance, et volonté. Car toute action d'un esprit demande posse, scire, velle. L'essence primitive de toute substance consiste dans la force; c'est cette force en Dieu qui fait que Dieu est necessairement, et que tout ce qui est en doit emaner." Carta a Morell, 29 de septiembre de 1698, Grua 139.
} 
por la razón, es decir, por ser las mejores" "44. La 'serie de posibilidades actuales' es la serie de las sustancias que, sin dejar de ser un agregado, constituye el mundo ${ }^{45}$.

Esto muestra que la emanación, que tiene su origen en Dios, produce las sustancias, ${ }^{46}$ los cuerpos orgánicos ${ }^{47}$ y toda la serie de cosas (series rerum) de los agregados. Muestra que la emanación expresa toda clase de fuerza que surge de la mente de Dios y expresa lo mejor, una fuerza que puede ser observada tanto en las sustancias como en los cuerpos. Precisamente esto vuelve a llevarnos al problema alma-cuerpo: la armonía preestablecida es asegurada por esta emanación continua, pues las diversas entidades emanadas están unidas por medio de la representatio como una concatenación ontológica y epistemológica, por medio del perspectivismo que determina la representación, y por medio de una potencialidad activa que alcanza hasta la causalidad desde el actus purus de Dios y el principio de lo mejor por medio de la finalidad de las mónadas. Ahora bien, esas transformaciones y representaciones no permiten ningún tipo de reduccionismo. Esto es lo que caracteriza la riqueza del idealismo leibniziano; pero al mismo tiempo se muestran aquí los presupuestos metafísicos sobre Dios y el mundo, sobre los que descansa esta solución al problema de la relación alma-cuerpo.

\section{Viejas respuestas, problemas nuevos}

¿En qué consiste entonces le significado de la respuesta leibniziana para la formulación actual del problema de la relación alma-cuerpo? El trasfondo ha cambiado radicalmente desde entonces, en parte a causa de los nuevos resultados científicos, que destruyen los principios leibnizianos de razón suficiente y continuidad. Nuestro concepto de materia es considerablemente más rico que

\footnotetext{
44 „Series haec actualium possibilium seu Mundus emanat ex Deo non necessario, alioqui aliae series non essent possibiles, sed tamen certa et determinata ratione, majoris scilicet boni." Discusión con Gabriel Wagner, 3 de marzo de 1698, Grua 396.

45 „Mundus vero est aggregatum plurium substantiarum“, Discusión con Gabriel Wagner, 3 de marzo de 1698, Grua 396.

${ }^{46}$ "la supreme substance, dont toutes les autres ne sont que des emanations et des imitations", Remarque sur ...Characteriticks of Men, Anexo a la carta a Coste del 30 de mayo de 1712, GP III 429ss.

${ }^{47}$ „Et rien ne pourra détruire tous les organes de cette substance, estant essentiel à la matiere d'estre organique et artificieuse partout, parce qu'elle est l'Effect et l'emanation continuelle d'une souveraine intelligence", Carta a Bassange, 1696, GP III 122.
} 
el del siglo XVIII; por eso no vacilamos lo más mínimo en considerar la energía como algo que -siguiendo a Einstein- posee un equivalente en masa. Conocemos muchos detalles del orden interno de un organismo hasta el análisis del genoma, y diariamente nos presenta la neurobiología resultados que son mucho más sutiles de lo que Leibniz se pudo imaginar nunca. Aún más, los problemas mismos se han transformado. Nadie intenta desarrollar una teoría acerca de la libertad de elección divina respecto a los mundos posibles, nadie busca una aclaración del paralelismo alma-cuerpo por medio de la armonía preestablecida como resultado de esa elección divina; y nadie querría pretender que todo el mundo sea orgánico. ¿Qué podemos aprender entonces de Leibniz?

Incluso cuando el horizonte se haya transformado, el problema nuclear permanece, a saber: ¿cómo es posible la libertad humana? ¿Cómo podemos explicar la relación alma-cuerpo? ¿Cómo podemos entender la conexión entre materia, organismo, datos neurobiológicos, hechos psicológicos y el pensamiento?

Presentemos en primer lugar esquemáticamente una tesis que es defendida con frecuencia: los neurofisiólogos explican que los filósofos deberían aprender que la voluntad libre es una absoluta ilusión, ya que esto ha sido probado claramente por experimentos: Cuando alguien cree levantar voluntariamente su brazo, lo que sucede es que el centro de su cerebro responsable del movimiento de su brazo, se vuelve activo algunas milésimas de segundos antes de que ese centro en el que solemos localizar las decisiones muestre una actividad. Por lo tanto, concluyen, tendríamos que aprender que no existe nada más que una serie de procesos electrónicos en el cerebro y que calificar a los mismos como libertad de la voluntad, incluso debido a su secuencia temporal, es una ilusión: No sólo Leibniz estaría por ello en un error cuando explica que Virgilio se equivoca al afirmar: "La mente mueve la masa, y se mezcla con todo el cuerpo". ${ }^{48}$ Ambos errores, el de Leibniz y el de Virgilio, son mucho más profundos, pues no hay ninguna mente. Lo que tenemos delante de nosotros es a lo sumo materialismo en toda su extensión. Pero esta tesis puede refutarse con ayuda de argumentos leibnizianos:

El primero de todos es que el modelo teórico de la neurobiología es hasta la fecha demasiado simple; pero su desarrollo subsiguiente es una cuestión de las ciencias empíricas, en todo caso, de la teoría de la ciencia, o, en lenguaje

48 „Mens agitat molem, et magno se corpore miscet“. Teodicea, Discurso Preliminar $₫ 8$, en Virgilio Eneida. 
leibniziano, de las conexiones internas dentro de la Scientia generalis. No continúo refiriéndome a él aquí porque no tiene una relación inmediata con el problema de la relación alma-cuerpo.

En segundo lugar, detrás de las apreciaciones actuales hay un error categorial: la libertad se basa en una elección racional entre alternativas previamente valoradas, y no en condiciones electroquímicas; estas condiciones pueden estar dadas simultáneamente con un phenomenon bene fundatum, pero su sentido, su semántica es completamente diferente de un estado fenoménico. Así, por ejemplo, las posibilidades representan alternativas; pero las posibilidades no son hechos, no pertenecen a este mundo como estados de cosas. Si existe un estado del cerebro que expresa una posibilidad, porque pensamos algo como una posibilidad, entonces esto es una cuestión que le compete a la semántica, y, como sabemos por Searle, la semántica no puede reducirse a la sintaxis, mientras que sólo reglas puramente formales, sintácticas pueden ser presentadas en la estructura de un estado electroquímico. Ahora bien, los neurobiólogos se defenderían en este punto aduciendo que la percepción de un estado de hechos está localizada en una región del cerebro diferente a aquella en la que se encuentra una mera posibilidad. Cierto, -pero para poder hacer esta distinción no sólo se requiere un conocimiento o una capacidad de discernir en sentido espacial, sino entre realidad existente y pura posibilidad; y esto ya no es una cuestión de neurología, sino de epistemología. En Leibniz aparece esto en la problemática de los conceptos primitivos como ideas atómicas, indescomponibles e indefinibles (como, por ejemplo, el concepto de la existencia y de la posibilidad), las cuales, para decirlo con Leibniz, pueden ser comprendidas por sí mismas: contienen en cierta medida su propia semántica. Aun cuando hoy día no consideremos válidos semejantes prima possibilia, estos indican el precio metafísico que tenemos que estar dispuestos a pagar, para conseguir una solución al problema de la relación alma-cuerpo.

$\mathrm{Al}$ rechazar hoy los prima possibilia de Leibniz, resta sin embargo la cuestión de cómo podemos entender conceptos como existencia, posibilidad, esencia, identidad o semejanza: Puesto que no podemos obtenerlos por medio de abstracción, tienen que ser presupuestos como forma prioritaria de pensamiento a la manera kantiana. El logicismo leibniziano-kantiano, a partir del que pueden reducirse objetos matemáticos a la lógica, apenas encuentra hoy día defensores, pero su trasfondo ontológico de un platonismo según el cual las estructuras matemáticas existen independientemente de la mente humana sigue siendo compartido por algunos eminentes científicos como Penrose. Incluso aun cuando no estemos dispuestos a aceptar ni ideas innatas ni formas a priori, desde que Chomsky 
fracasara en la búsqueda de universales lingüísticos, no podemos explicar cómo es posible que en teorías científico-naturales encuentren aplicación formalismos matemáticos sin aceptar al mismo tiempo una especie de platonismo mínimo, un platonismo con una "p" minúscula -como lo ha denominado Paul Bernays. Todo esto muestra que la ontología neurobiológica no es lo suficientemente rica: necesita ser completada al menos con una semántica y un platonismo mínimo. Ahora bien, tan pronto como estamos dispuestos a ello, nos encontramos confrontados con la problemática de la mente o del alma, puesto que ni la matemática ni la semántica serían explicables sólo en el nivel de los cuerpos y de la materia.

Tercero, Leibniz transforma el cogito cartesiano y con ello aclara que debemos partir de nuestra propia experiencia como un individuo, como un yo unitario. Para hablar de los otros como mónadas, atribuirles individualidad y capacidad de pensar, presupone, por una parte, el concepto de analogía como forma de pensar, por otra parte, la existencia de otros cuerpos fenoménicos -no como amasijos de materia sino como individuos. Pero una forma de unidad tal no se encuentra en la materia, a no ser que yo se la atribuya; y no existiría nada que fuera responsable de sus acciones, si no existiera ninguna persona. Esto significa que los derechos humanos -o los principios leibnizianos de la justicia, esto es, honeste vivere, neminem laedere, suum cuique tribuere- presuponen la unidad de la persona, que no está constituida de ninguna manera por la materia.

Cuarto, Leibniz creía que el mundo de las mónadas era un mundo lleno de vida. En esto ya no le seguiríamos hoy, pero nos encontramos ante el problema de explicar cómo en el comienzo de nuestro universo pudieron originarse, a partir de un plasma casi homogéneo y altamente energético, primero partículas, luego átomos y moléculas, y finalmente macromoléculas altamente complejas con capacidad de autoreproducción, las cuales, unidas a agregados bióticos, desarrollaron un sistema nervioso y, por último, llegaron a tener estados mentales. Al igual que Aristóteles, Leibniz incluyó en el mismo grupo a seres humanos, animales y plantas, atribuyéndoles percepciones, mientras que se distanció de Aristóteles al incluir en el mismo grupo a Dios y excluir la materia como fenoménica. Hoy ha cambiado el paradigma dominante y, por regla general, nos encontramos totalmente del lado opuesto: no Dios, sino la materia es escogida como punto de partida, mientras que prefiere excluirse el otro extremo, la mente, por no hablar de Dios. Pero precisamente lo mismo que Leibniz tenía que explicar qué eran las sustancias corporales en conexión con los cuerpos materiales como phenomena bene fundata, nos encontramos hoy con la dificultad de que una consideración que parte de una explosión originaria sería absolutamente incompleta, si 
excluyese la mente como algo reducible a la materia, tal y como se ha propuesto el materialismo eliminativo.

En todo caso, el problema mente-cuerpo de hoy en día no es sino un paso en el desarrollo en cuestión desde el 'Big Bang'. Esto deja claro que hemos de introducir un concepto que nos permita describir (y quizás no explicar) y salvar el vacío entre un nivel y el siguiente en tanto emergencia de las nuevas propiedades en el nivel superior. Esto ha de ser emprendido de manera no reductivista, puesto que no hay posibilidad de predecir el desarrollo aquí en liza. Recordemos que Leibniz, a fin de solventar el problema mente-cuerpo por medio de la armonía pre-establecida, se vio forzado a introducir un tercer nivel, esto es, la región de las ideas en la mente divina, y a enriquecerlo por medio de una actividad dentro de dicha mente. Por lo tanto, en la actualidad el resultado no puede consistir en una reproducción de la doctrina aristotélica de las capas ontológicas únicamente, sino que se requiere la idea aristotélico-leibniziana de una energeia o potencialidad que impulsa los estados, de modo que esta potencialidad crea absolutamente nuevas clases de individuos que tienen absolutamente nuevas propiedades, como se sabe del caso especial que es la evolución, y que acaba en individuos con consciencia.

Probablemente no es de ayuda alguna el creer que ya en el primer estado plasmático a altas temperaturas se incluyeran todas las consecuencias, ya que no existe principio de razón suficiente detrás (incluso y aún en el caso de que la ley de desarrollo interno se tenga en cuenta en la discusión contemporánea como principio antrópico). Pero a fin de cuentas todo esto viene a significar que hemos de buscar una nueva y más apropiada respuesta que debería contemplar cuál es la manera en que Leibniz la intentaría contestar.

Las últimas reflexiones muestran que el problema de la relación alma-cuerpo, hoy lo mismo que para Leibniz, no puede considerarse únicamente como un problema de la mente humana y del cuerpo humano, sino como problema parcial en el desarrollo del universo en expansión desde la denominada explosión originaria. Solo en un marco semejante lo suficientemente amplio pueden concebirse las citadas conexiones, porque el problema de la relación alma-cuerpo ya para la tradición no se podía clasificar de otra manera. Con ello, se muestra que tiene que ser desarrollado un modelo que nos permita describir y quizá también explicar cómo puede entenderse el camino desde el comienzo esbozado, a través de los distintos niveles, como una emergencia de las respectivas nuevas cualidades sobre las respectivas del nivel más elevado. Esto debe suceder de una manera no reduccionista, porque no hay ninguna posibilidad de predecir las nuevas cualidades en el nivel precedente, $o$ 
en el nivel ontológicamente inferior, por decirlo en términos aristotélicos. Con ello recordamos que Leibniz, para solucionar el problema de la relación alma-cuerpo por medio de su armonía preestablecida, se vio obligado a aceptar como nivel más elevado la regio idearum del pensamiento divino y, además, a enriquecerlo con una actividad de la mente divina. De acuerdo con esto, no se puede volver a aceptar hoy simplemente la doctrina aristotélica de las capas ontológicas, sino que se requiere una concepción leibniziano-aristotélica de una energeia o potencialidad, la cual impulse las situaciones - ahora bien, de abajo hacia arriba -, de forma que esa potencialidad absoluta pueda producir nuevas especies de individuos con cualidades absolutamente nuevas, como es conocido a partir de las teorías evolutivas, en las que al final se consigue producir el individuo con conciencia. Probablemente no nos sea de mucha ayuda aceptar, en el sentido del principio antrópico, que el primer plasma altamente energético ya contiene en sí todos sus estados subsiguientes -tal y como Leibniz lo aceptó para cada mónada-, porque el principio de razón suficiente en su radio de alcance se nos presenta como limitado desde nuestra comprensión actual. En efecto, todo esto muestra cuán alejados estamos de una respuesta satisfactoria.

Si echamos una mirada retrospectiva al desarrollo del problema de la relación alma-cuerpo en Leibniz y hoy día, parece claro que se trata de dos formas de acceso muy diferentes, que descansan en modos de plantear cuestiones muy diversas. Leibniz intentó desarrollar un "sistema" como una "hipótesis" que conducía desde la emanación de Dios a través de los individuos y sus percepciones hasta el mundo percibido, mientras que hoy día partimos de un fundamento físico para, a partir de allí, llegar en una emergencia a la mente a través de biosistemas. Ambos puntos de vista son perspectivas externas sub specie aeternitatis, pues ambos admiten un topos externo: Leibniz intenta ver a Dios en los mapas, mientras que nosotros intentamos llegar detrás del enigma de la evolución desde una postura de observador. Los principios que se presuponen en ambos casos son absolutamente distintos. Leibniz desarrolla su representación sobre la base de la lógica y del principio de razón suficiente que, al mismo tiempo, es también el principio de causalidad suficiente; por ello aparecen vinculadas causas y principios, de forma que, lo que él desarrolla es válido tanto para las mónadas como para los cuerpos. Con lo cual viene a relacionar esto con una perspectiva teleológica, en tanto que incluye en el principio de razón suficiente el principio de lo mejor como un principio moral para regir las acciones divinas y humanas. Todo esto se diferencia radicalmente de los presupuestos actuales: desde que la teoría cuántica ha dejado de ser discutible, denegamos al principio de razón suficiente su validez universal, y desde la Crítica del Juicio de Kant hemos dejado de buscar interpretaciones teleológicas de la naturaleza. Antes bien, lo que intentamos es diseñar una teoría de una nueva forma de 
desarrollo fuera o junto al desarrollo causal, como se ve, por ejemplo, en la relación de la teoría cuántica de campos y la física clásica, junto con las interpretaciones que aseguran la independencia de la conciencia; o en una perspectiva teorética procesual, que acopla con procesos de conciencia los procesos evolutivos finales debilitados; o en los modelos del caos determinista, de la evolución y de la autoorganización, ejemplos en los que la no predecible aparición de algo completamente nuevo, es posible seguida de selección o estabilización. Esta nueva forma de ver es tan poderosa que ha encontrado su camino en muchos campos científicos en los que era válido estructurar procesos temporales; ahora bien, hoy ha alcanzado en la forma de teorías de la emergencia al problema de la relación alma-cuerpo.

El esquema de la evolución es la nueva visión del mundo, que ha aparecido en el lugar de la visión causal mecanicista; y con ello se ha desplazado la cuestión con la que confrontarse científicamente. Ya no se trata de cómo llevó Dios la emanación al mundo, sino - en lugar de una solución top-down, una bottom-up - de cómo algo completamente nuevo pudo convertirse en real. También aquí aparece un notable paralelismo con Leibniz: para su teoría de la emanación necesitaba Leibniz una especie de fuerza (vis), que tenía que hacer su aparición como vis primitiva y fiat! Divino ante las posibilidades de la regio idearum, para entrar a formar parte como appetitus en las mónadas y como vis derivativa en la dinámica del mundo de los cuerpos, -en ambos casos combinada además con un tipo especial de potencialidad activa: este es en realidad el precio metafísico de la solución leibniziana. Si miramos a partir de aquí hacia las teorías de la emergencia, se muestra que precisan algo análogo, esto es, una especie de potencialidad dinámica. Ésta no puede ser de naturaleza teleológica, sino que ha de permanecer abierta hacia el futuro, impulsando a su vez la evolución de manera que, con las características de la emergencia, surjan nuevos niveles con nuevos objetos y nuevas cualidades, que revelen un tipo especial de necesidad. También aquí se presenta el problema, el problema de la sustancia corporal, pero ahora sólo como un problema botton-up: la prima materia es un apeiron anaximándrico de elevada energía, pero ¿cómo podemos introducir entonces orden por así decirlo contra la segunda ley de la termodinámica? ¿cómo puede llegar éste a la auto-organización y auto-reproducción de biosistemas y, finalmente, a la mente?

Mientras que Leibniz colocaba en el nivel superior el reino de las ideas y la actividad de la mente divina, nosotros tenemos que ampliar nuestro nivel inferior: el plasma lleno de energía no es de ninguna manera la materia tradicional -sobre todo tienen que ampliarse las potencialidades que determinan el universo en expansión con su dinámica interna. Todo esto suena a pura física, pero de hecho se trata de 
una metafísica de estados hipotéticos, que posibilitan la libertad en el nivel superior; quizá deberían por ello los científicos naturales denominar también a sus nuevos sistemas como hipótesis, tal y como lo hizo Leibniz. En cualquier caso, la teoría de la emergencia es una teoría metafísica, quizá fértil, si estamos dispuestos a sondear y analizar a la manera leibniziana sus presupuestos metafísicos.

Si el neurobiólogo tuviera razón con su supuesta demostración a favor de la no existencia de la libertad de la voluntad, ni su afirmación sería libre, ni la consecuencia de una reflexión, sino el resultado de un acto de habla o escritura que tuvo lugar antes de que comenzara a pensar. Si no aceptase esto, su propio modelo fracasaría. En cualquier caso, seguimos con nuevas preguntas y viejas respuestas -respuestas que no solucionan el problema actual, pero que nos muestran porqué algunas de las nuevas propuestas de solución son insostenibles y qué tipo de metafísica tiene que entrar en escena, para que pueda ser posible una solución hipotética.

Recibido: 1/10/2016

Aceptado: 21/10/2016

Este trabajo se encuentra bajo una licencia de Creative Commons ReconocimientoNoComercial-SinObraDerivada 4.0 Internacional 
